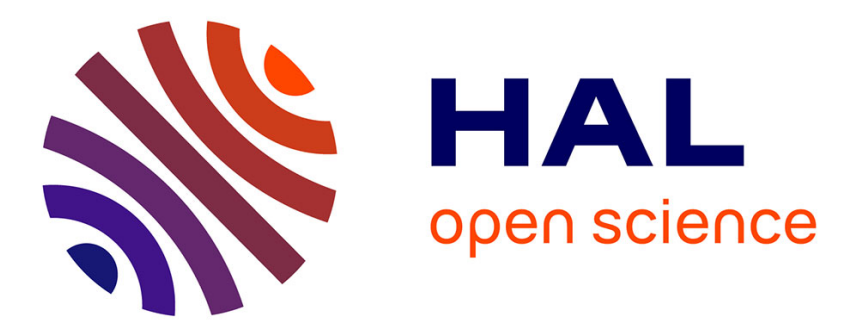

\title{
Revealing stiffening and brittling of chronic myelogenous leukemia hematopoietic primary cells through their temporal response to shear stress
}

B Laperrousaz, L Berguiga, F.E. Nicolini, C. Martinez-Torres, A. Arneodo, V. Maguer-Satta, Franck Emmanuel Nicolini, Françoise Argoul

\section{- To cite this version:}

B Laperrousaz, L Berguiga, F.E. Nicolini, C. Martinez-Torres, A. Arneodo, et al.. Revealing stiffening and brittling of chronic myelogenous leukemia hematopoietic primary cells through their temporal response to shear stress. Physical Biology, 2016, 13 (3), pp.06LT01 (1-6). 10.1088/14783975/13/3/03LT01 . hal-01343169

\author{
HAL Id: hal-01343169 \\ https://hal.science/hal-01343169
}

Submitted on 7 Jul 2016

HAL is a multi-disciplinary open access archive for the deposit and dissemination of scientific research documents, whether they are published or not. The documents may come from teaching and research institutions in France or abroad, or from public or private research centers.
L'archive ouverte pluridisciplinaire HAL, est destinée au dépôt et à la diffusion de documents scientifiques de niveau recherche, publiés ou non, émanant des établissements d'enseignement et de recherche français ou étrangers, des laboratoires publics ou privés.

\section{다(1)(2)}

Distributed under a Creative Commons Attribution - ShareAlikel 4.0 International 


\title{
Revealing stiffening and brittling of chronic myelogenous leukemia hematopoietic primary cells through their temporal response to shear stress
}

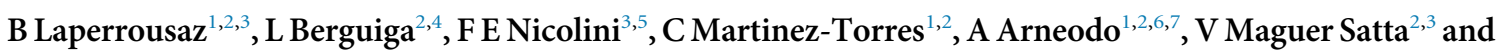 \\ F Argoul ${ }^{1,2,6,7}$ \\ 1 CNRS UMR5672, Laboratoire de Physique, Ecole Normale Supérieure de Lyon, 46 Allée d’Italie, 69007 Lyon, France \\ 2 Université de Lyon 1, 43 Boulevard du 11 Novembre 1918, 69100 Villeurbanne, France \\ 3 CNRS UMR5286, INSERM U1052, Centre de Recherche en Cancérologie de Lyon, 28 rue Laennec, 69008 Lyon, France \\ 4 CNRS UMR5270, Institut des Nanotechnologies de Lyon, INSA Lyon, 7 avenue Capelle, 69621 Viilleurbanne, France \\ Hospices Civils de Lyon, Hematology Department, CHLS, 165 Chemin du Grand Revoyet, 69495 Pierre Bénite, France \\ 6 CNRS UMR5798, Laboratoire Ondes et Matière d'Aquitaine, 351 cours de la Libération, 33405 Talence, France \\ Université de Bordeaux, Campus de Talence, 351 cours de la Libération, CS 10004, 33405 Talence, France
}

Keywords: viscoelasticity, cell mechanics, cancer stem cells, wavelet transform analysis, chronic myelogenous leukemia, primary cells

Supplementary material for this article is available online

\begin{abstract}
Cancer cell transformation is often accompanied by a modification of their viscoelastic properties. When capturing the stress-to-strain response of primary chronic myelogenous leukemia (CML) cells, from two data sets of CD34+ hematopoietic cells isolated from healthy and leukemic bone marrows, we show that the mean shear relaxation modulus increases upon cancer transformation. This stiffening of the cells comes along with local rupture events, detected as reinforced sharp local maxima of this modulus, suggesting that these cancer cells respond to a local mechanical stress by a cascade of local brittle failure events.
\end{abstract}

Cell mechanics is recognized to control critical cellular functions and to be altered in different human diseases including cancer [1-10]. As such, cell mechanics may help unravel the role of mechanical alterations in cell transformation processes and to quantify how malignant cells differ from healthy ones. Chronic Myelogenous Leukemia (CML) has been described as a myeloproliferative disorder that arises from a single reciprocal chromosomal translocation $\mathrm{t}(9 ; 22)$ leading to the formation of an oncogene (BCR-ABL) [11]. The bone marrow cell density considerably increases during this disease, suggesting that the leukemic hematopoietic cell deformability may also change [12]. The purpose of this letter is to show that these cells not only become stiffer and more elastic upon transformation but that their ability to sustain a deformation is dramatically changed, as evidenced by enhanced local rupture events indicating a higher brittleness.

Previous studies of cancer cell elasticity [2, 3] have shown that, depending on the cell types and the context, the stiffness of cancer cells may increase $[6,10]$ or decrease [13] and their Young modulus distributions may spread or shrink [5], when compared to healthy counterparts. Recent advances in nanotechnologies have paved the way to single-cell mechanics studies. Atomic force microscopy (AFM) is one of the local probe method that has proved efficient to measure single living cell stiffness [14-20]. However, very few studies consider that, during their deformation, living cells remodel their shape in real time by viscous or plastic effects that take over a part of the input energy. Such an assumption requires that the characterization of the cell response be not simply restricted to a single elastic parameter (Young's modulus) [21]. One of the major difficulties in capturing the relevant parameters that could describe the stress-to-strain response of a living cell comes from our lack of realistic models. As soon as we try to restrict the dynamical response of a living cell to a simple viscoelastic model, we lose an important information, namely its local and dynamical (active) adaptation to a given deformation. We take the opposite view in this study. Without hypothesizing 
any model, we adapt sophisticated space-scale wavelet-based analyzing tools to capture, as accurately as possible, the dynamical signature of the force indentation curves (FICs) recorded during a living cell AFM indentation. Prior to this study, this space-scale analysis was successfully applied to characterize AFM images of rough surfaces [22] and mechanical footprints of isolated plant cell walls [23], to image living cells via diffraction phase microscopy [24, 25] and to capture the passive micro-rheology of living adherent cells with AFM [26]. In the context of the present study, this characterization provides evidence for local rupture events (brittle failures) that overwhelm the background noise in the pathological case (CML). These brittle failures could constitute a new marker for cell resilience to deformation in cancer.

The nano-indentation of living cells was performed with an atomic force microscope (AFM) on single hematopoietic purified CD34+ cells from CML patient and healthy donor bone marrows ( $>95 \%$ purity). Hematopoietic cells were immobilized on fibronectin-coated coverslips to avoid them slipping away from under the cantilever tip when performing the nano-indentation. We believe that this fibronectin adhesion assay is relevant because it is rather representative of physiological conditions observed in the human bone marrow that hosts the hematopoietic stem cell niche. Actually these cells are not in suspension in their niche but actively adhere to stromal cells and to proteins of the extracellular matrix. The technical description of the cell preparation, the AFM measurement and the force indentation curve (FIC) analysis is thoroughly described in the Supplementary data $^{8}$. We focus more precisely on the local curvature of the FICs (figure 1), that is characteristic of the shear relaxation modulus $G_{r}(Z)$ of the tested sample (equation S7 in (see footnote 8)). We computed $G_{r}(Z)$ as a smoothed second-order derivative of the force curve $F: T_{g^{(2)}}[F](Z, s)$ (equation S14, S15 in (see footnote 8$)$ and [23]). The variations of $G_{r}(Z)$, for a fixed scale $s$ value (corresponding to an analyzing wavelet width of $60 \mathrm{~nm}$ ), are illustrated in figures $1(\mathrm{c})$, (f). This width of the second derivative of a Gaussian $g^{(2)}$ analyzing wavelet (see footnote 8) was optimized to smooth down the background noise. This smoothing is illustrated on the force curves in figures 1(b), (e) that were filtered by a Gaussian function $g^{(0)}$ of the same width (red lines). We note that local peaks (extrema) in $G_{r}(Z)$ can be clearly distinguished from the background noise for CML cells (figure 1(c)). Interestingly, none (or few) of these peaks can be detected for the healthy cells (figure 1(f)). The number $N_{p}$ of maxima for a given FIC and their mean amplitude $G_{<p>}$ along the $G_{r}(Z)$ profile will be used to distinguish healthy from cancer cells in the next paragraph. We also notice that the left FIC (figure 1(a)) presents three regimes, a

\footnotetext{
${ }^{8}$ See Supplementary Material file for a more detailed description of materials and methods, cell culture and analysing tools.
}

very slow increase of $F$, followed by a faster increase after $500 \mathrm{~nm}$ indentation depth, and finally a much sharper increase beyond $800 \mathrm{~nm}$ indentation. It is thus quite impossible to extract a single shear modulus from this curve, that would require fitting this curve by a single parabola, as predicted by a purely elastic Sneddon model [27]. Indeed, the slow increase of $F$ in the first regime is likely to be heavily influenced by the membrane and its outer sheath (gangliosides in the case of hematopoietic stem cells), whereas the third regime at the end of the force curve appears to be sensitive to the nuclear area. This is why we chose to focus on the middle part of this curve and fit the smoothed red curve by a parabola (plotted as a blue line in figures 1(a), (b)), from which we get some representative estimate of a mean shear relaxation modulus $\left(G_{m}\right)$. The story of the indentation is completely different for the cell from an healthy donor (figure 1(d)), where $F$ increases smoothly and can be better approximated by a parabolic shape (blue curve) with a single coefficient which will be considered as a mean shear relaxation modulus $\left(G_{m}\right)$. In addition, the dissipation loss ratio is computed from the work integral of the approach curve $W_{a}$ (equation S2 in (see footnote 8)) and of the retract curve $W_{r}$ (equation S3 in (see footnote 8)) as $D_{r}=\left(W_{a}+W_{r}\right) / W_{a}$ (equation S4 in (see footnote 8)).

Figure 2 summarizes the distribution of all the parameters: $G_{m}, G_{<p>}, D_{r}$ and $N_{p}$, computed from two large sets of single cell FICs collected from $5 \mathrm{CML}$ patients (red - 1302 force curves -51 cells) and 5 healthy donors (blue - 1672 force curves - 60 cells). Figure 2(a) shows the histograms of $G_{m}$ values whose medians clearly indicate that the CML cells are stiffer $\left(G_{m}=705 \mathrm{~Pa}\right)$ than the healthy ones $(360 \mathrm{~Pa})$. We note also that in the healthy case, $50 \%$ of FICs bear a mean shear relaxation modulus smaller than $350 \mathrm{~Pa}$, whereas in the cancer case the histogram of $G_{m}$ values is much wider and has a maximum around $700 \mathrm{~Pa}$, meaning that the transformed hematopoietic cells in CML are about twice stiffer on average than their healthy counterpart, confirming previous observations in other leukemia $[1,4,10]$. Our analysis of the local curvature of the force curves, via the detection of their local maxima, provides an important clue to the understanding of how these cancer cells can adapt to a deformation. The distribution of the mean value of the maxima height $G_{<p>}$ is illustrated in figure 2(b). These local values of the shear relaxation moduli $G_{<p>}$ (corresponding to the peaks of $G_{r}$ ) are much larger than $G_{m}$ averaged over the indentation curve (figure 2(a)); they indeed describe very local events occurring at scales below $60 \mathrm{~nm}$ (the size of the analyzing wavelet). The histogram of $G_{<p>}$ values is not only shifted to larger values in the pathological case (with a median $=24 \mathrm{kPa}$ slightly larger than $19 \mathrm{kPa}$ in the healthy case), but is also more asymmetric, spreading to twice larger values than for the healthy case. The comparison of the distributions of dissipation loss ratio $D_{r}$ (figure 2(c)) leads to two main observations: 

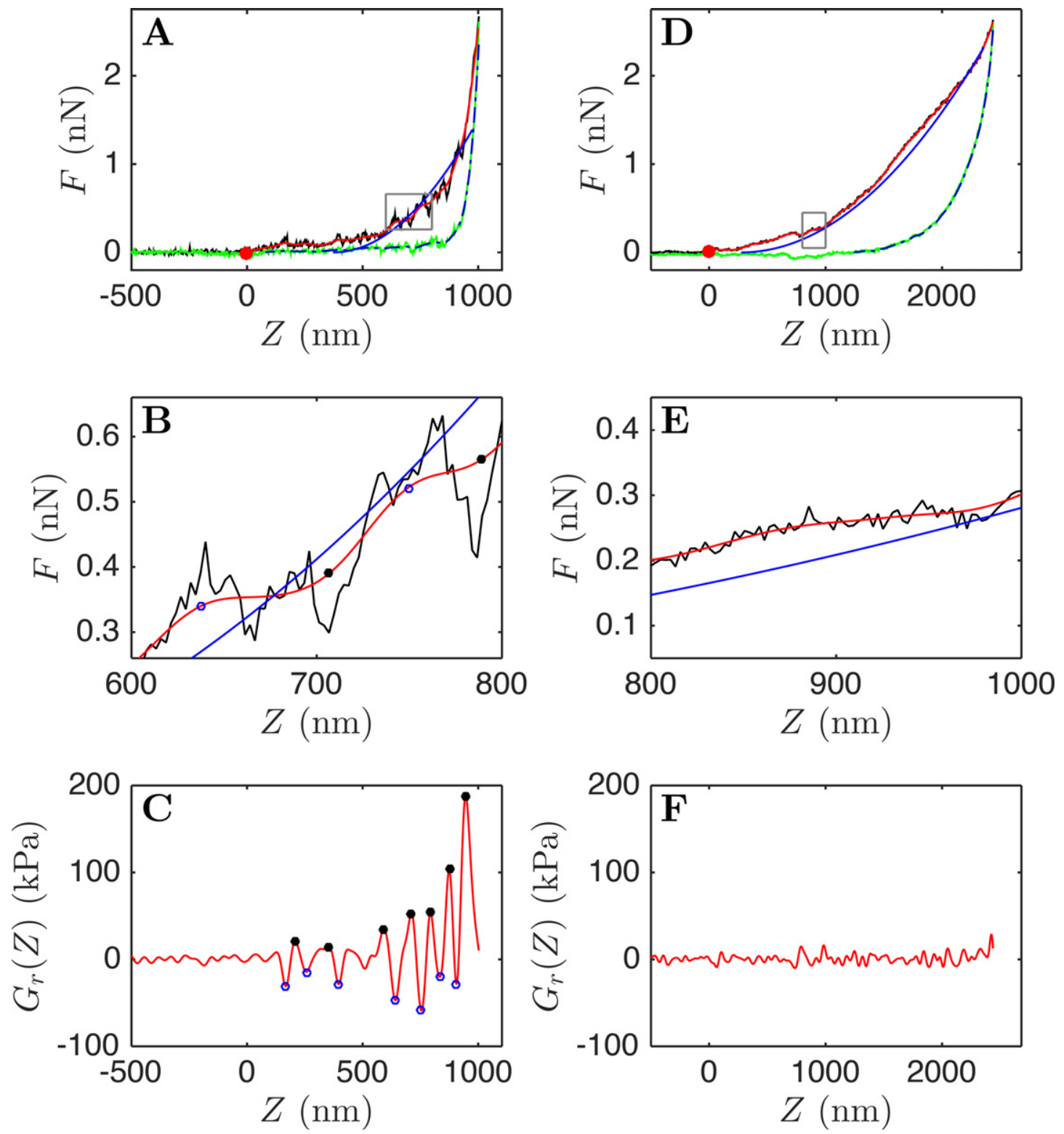

Figure 1. Illustration of FICs and shear relaxation moduli $G_{r}(Z)$ (see (see footnote 8)) from two CD34+ cells selected from a CML patient (a) (c) and a normal donor (d) (f).(a), (d) $F(Z)$ curves showing both the approach (black) and retract (green) curves. The red curves correspond to some smoothing of the original FICs with a low pass filtering Gaussian $g^{(0)}$ (see footnote 8). The blue curves are parabolic parametrizations of the FICs, that match their local derivative in their middle part, the pre factor giving some estimate of the mean shear relaxation modulus $G_{m}$ (see text). The dashed dark grey curves outline the part of the retract curves which is used for the computation of the recovered work after releasing the stress. (b), (e) Zooms of (a), (d) highlighting the presence of a large number of rupture events in the CML case. (c), (f) $G_{r}(Z)$ computed with a second derivative of a Gaussian with the same width as the Gaussian filter used in (a), (d). The black dots (resp. blue circles) correspond to the local maxima (resp. minima) of $G_{r}(Z)$ selected according the criteria that $\Delta G_{r}(Z)$ between two neighbouring extrema $>25 \mathrm{kPa}$. These local extrema positions are reported on the FIC zooms in (b), (e).

the cancer cells appear less viscous (median $D_{r}=0.57$ ) than the healthy ones (median $D_{r}=0.63$ ), and there is also a significant fraction of FICs corresponding to low dissipation loss ratio $D_{r}$ in the $[0.2 ; 0.4]$ interval for cancer cells that has no counterpart in healthy cells. Importantly, the statistical sampling of the force curves with detectable local maxima is not the same in cancer ( $87 \%$ of the FICs) and healthy (64\% of the FICs) cells. In the former, not only the amplitude of these local shear relaxation modulus maxima increases but their probability of occurrence also increases, whereas the length of the indentation curves rather tends to decrease (by a factor two on average). This would therefore give a reduction of the density of these local failure events by a factor of three from cancer to healthy cells. This conclusion is corroborated by the percentage of FICs showing a given number $N_{p}$ [from 0 to 10] of $G_{r}$ local maxima (figure $2 D$ ). More than $50 \%$ of the force curves from healthy donors do not contain any failure event or at the very most a single event, as compared to only $25 \%$ of the force curves from CML patients. This means that the excess of 


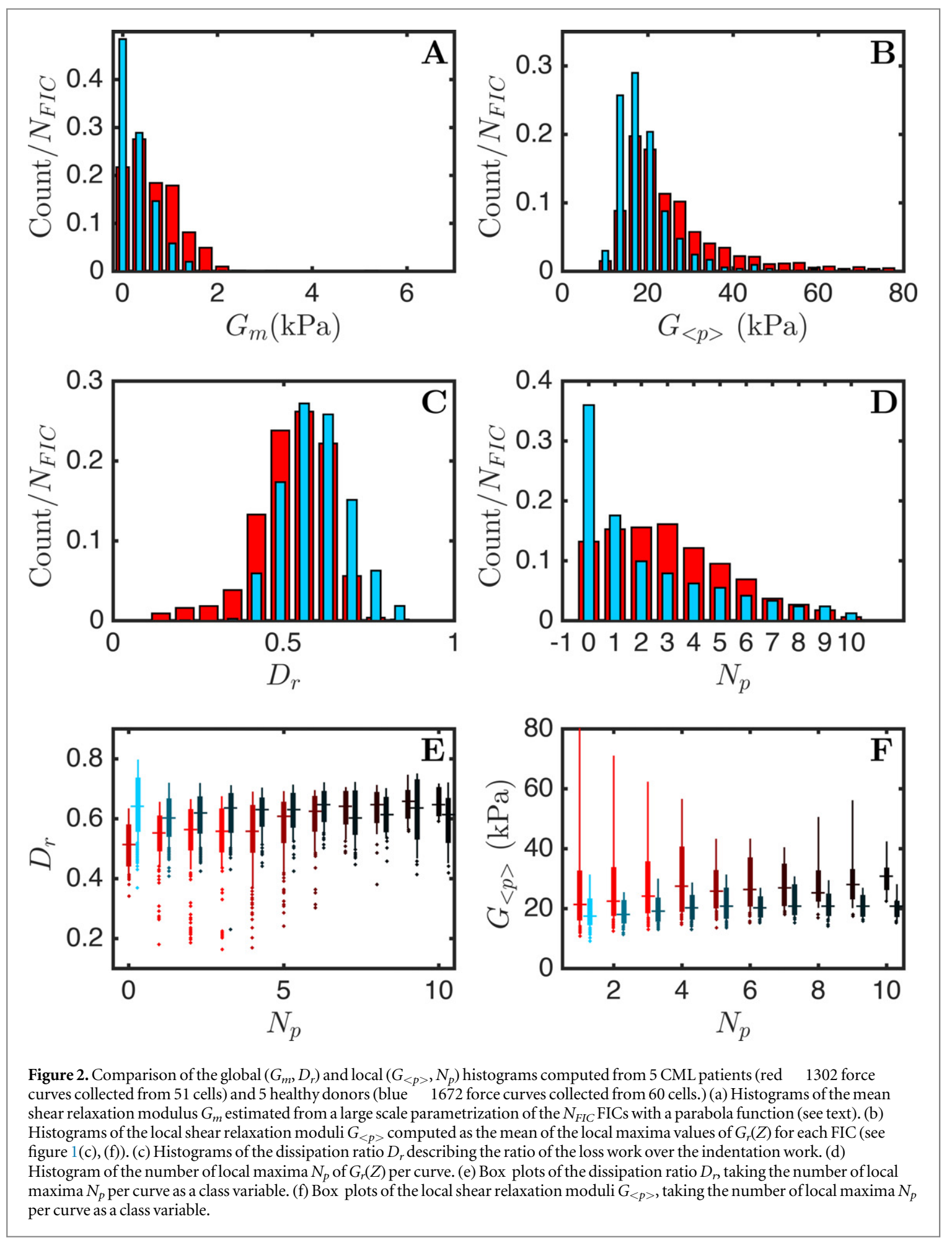

failure events observed in a majority of cancer FICs is a clear signature of the mechanical transformation of these cells. It is also quite informative to look for both cases at the two quantities $G_{<p>}$ and $D_{r}$ on each subset of FICs with a given number of $G_{r}$ maxima. The boxplots of figure 2(e), (f) show for each subset, the median (horizontal line), the quartiles (25\% and $75 \%$ ) as the limit of the vertical boxes, and the $10 \%$ and $90 \%$ percentiles as the edge of the thinner lines. The outliers are plotted as single dots. The color of the box-plots is proportional to the percentage of FICs used to compute these boxes, as given by the histogram in figure 2(d). We note that the distinction of the $D_{r}$ values between cancer and healthy cells is more prominent on the FICs with a number of peaks $N_{p}$ in the range of 2 to 4 , corresponding to $44 \%$ of the FICs in cancer cells and to only $24 \%$ in healthy cells. The outliers with a much lower $D_{r}$ are also much more distant from the quartiles boxes in cancer than in healthy cells. Finally, when comparing the amplitude of the local $G_{r}$ 
maxima, averaged over the different peaks on each FIC (figure 2(f)), we notice again an amplification of these maxima amplitude in the cancer case, and the reported box-plots show that the greater amplification also occurs for FICs with $N_{p}$ ranging from 2 to 4 . This is a strong indication that CML cells have the propensity to become (at least locally) stiffer and less viscous than healthy cells. Let us emphasise that the extreme variability in the viscoelastic properties of cancer cells obtained from the analysis of our overall sample of 1320 FICs from 51 CML cells is quite representative of the results obtained for each individual CML patient.

This study suggests a possible recognition of cancer transformation in leukemia through local singular events that would sign local failure of the cell cytoskeleton cohesion. Given that the cytoskeletal elasticity is elaborated by a complex interplay between active and cross-linked biopolymer networks [28-30], the quick adaptation of these cross-links is essential for the ability of living cells to sustain local shear stress without failure. If these cross-links are tight and less active, elastic behaviour of these networks can become locally nonlinear, making the cell looking much stiffer upon applied stress [30, 31]. In that case a brittle failure involving a collective unbinding of cross-links may occur, leading to a catastrophic loss of network integrity [32]. In CML, the BCR-ABL fusion gene is detected in up to $95 \%$ of CML patients and gets translated into an oncoprotein $\mathrm{p} 210^{B C R / A B L}$ that has increased tyrosine kinase (TK) activity and increased binding to the actin cytoskeleton [33] compared to the p145 Abelson protein. The interaction of $\mathrm{p} 210^{B C R / A B L}$ with F-actin was shown to induce its redistribution into punctate, juxtanuclear aggregates [34]. In addition, the interaction of $\mathrm{p} 210^{B C R / A B L}$ with other actin-cross linking proteins such as $\alpha$-actinin and fimbrin (by similitude with the c-Abl F-actin-binding domain) could modify the actin cytoskeleton mechanosensitivity properties [34, 35]. To summarise, we have shown in this study that upon elastic stiffening, CML CD34+ hematopoietic cells respond very abruptly to a local mechanical stress, by a cascade of local brittle failure events, whereas healthy CD34+ hematopoietic cells are able to maintain their network integrity by a rapid network adaptation (ductile failure). These results open new perspectives in the understanding of how leukaemia cells can withstand mechanical constraints exerted by their microenvironment to promote their survival and drug escape [36-39].

\section{Acknowledgments}

This study was funded by Agence Nationale de la Recherche ANR-10-BLAN-1516, INSERM (Plan Cancer 2012 01-84862), CNRS, Novartis, Ligue Nationale contre le Cancer (Saone et Loire) and Cent pour Cent la Vie. $\mathrm{BL}$ is the recipient of a $\mathrm{PhD}$ fellowship from the
French government and by the Ligue Nationale contre le Cancer, CMT PhD fellowship was partly supported by CONACyT, Mexico.

\section{References}

[1] Rosenbluth M J, Lam W A and Fletcher D A 2006 Biophys. J. 90 29943003

[2] Suresh S 2007 Acta Bio. 341338

[3] Brunner C, Niendorf A and Käs J A 2009 Soft Matter 5 21718

[4] Cai X, Xing X, Cai J, Chen Q, Wu S and Huang F 2010 Micron 4125762

[5] Lekka M2012 Nat. Nanotechnol. 76912

[6] Ketene A N, Schmelz E M, Roberts P C and Agah M 2012 Nanomedicine: Nanotechnol. Biol. Med.893 102

[7] Byun Setal 2013 Proc. Natl Acad. Sci. 11075805

[8] Wang B, Lançon P, Bienvenu C, Vierling P, Di Giorgio C and Bossis G 2013 Micron 4428797

[9] Rother J, Nöding H, Mey I and Janshoff A 2014 Open Biol. 4 140046

[10] Zheng Y, Wen J, Nguyen J, Cachia M A, Wang C and Sun Y 2015 Sci. Rep. 57613

[11] Pasternak G, Hochhaus A, Schultheis B and Hehlmann R 1998 J. Cancer Res. Clin. Oncol. 12464360

[12] Jones D 2010 Neoplastic Hematolopathology: Experimental and Clinical Approches (New York, NY: Humana)

[13] Sokolov I 2007 Atomic force microscopy in cancer cell research Cancer Nanotechnology (Valencia, CA: American Scientific) pp 17

[14] Radmacher M, Tillmann R W and Gaub H E 1993 Biophys. J. 6473542

[15] Radmacher M 2002 Methods in Cell Biology 686790

[16] Jena B P and Horber J K H 2002 Atomic Force Microscopy in Cell Biology (San Diego, CA: Elsevier)

[17] Hecht F M, Rheinlaender J, Schierbaum N, Goldmann W H, Fabry B and Schäffer T E 2015 Soft Matter 11458491

[18] Cartagena Rivera A X, Wang W H, Geahlen R L and Raman A 2015 Sci. Rep. 511692

[19] Dokukin M and Sokolov I 2015 Scientific Report 512630

[20] Abidine Y, Laurent V M, Michel R, Duperray A and Verdier C 2015 Eur. Phys. J. Plus 130202

[21] Kiessling T R, Herrera M, Nnetu K D, Balzer E M, Girvan M, Fritsch A W, Martin S S, Kas J A and Losert W 2013 Eur. Biophys. J. 4238394

[22] Roland T, Khalil A, Tanenbaum A, Berguiga L, Delichère $P$, Bonneviot L, Elezgaray J, Arneodo A and Argoul F 2009 Surf. Sci. 603330720

[23] Digiuni S, Berne Dedieu A, Martinez Torres C, Szecsi J, Bendahmane M, Arneodo A and Argoul F 2015 Biophys. J. 108 223548

[24] Martinez Torres C, Berguiga L, Streppa L, Boyer Provera E, Schaeffer L, Elezgaray J, Arneodo A and Argoul F 2014 J. Biomed. Opt. 19036007

[25] Martinez Torres C, Laperrousaz B, Berguiga L, Boyer Provera E, Elezgaray J, Nicolini F E, Maguer Satta V, Arneodo A and Argoul F 2015 J. Biomed. Opt. 20096005

[26] Martinez Torres C, Arneodo A, Streppa L, Argoul P and Argoul F 2016 Appl. Phys. Lett. 106034102

[27] Sneddon I N 1965 Int. J. Eng. Sci. 34757

[28] Khan S M, Ali R, Asi N and Molloy J E 2012 Commun. Integr. Biol. 53942

[29] Huber F, Schnauß J, Ronicke S, Rauch P, Muller K, Futterer C and Kas J 2013 Adv. Phys. 62112

[30] Jensen M H, Morris E J and Weitz D A 2015 Biochim. Biophys. Acta 1853303842

[31] Ehrlicher A J, Krishnan R, Guo M, Bidan CM, Weitz D A and Pollak M R 2015 Proc. Natl Acad. Sci. 112661924

[32] Sadhukhan P, Schumann O and Heussinger C 2014 Eur. Phys J. E 3758

[33] McWhirter J R and Wang J Y J 1991 Mol. Cell. Biol. 11 155365 
[34] McWhirter J R and Wang JY 1993 The EMBO Journal 12 153346

[35] Goldman J M and Melo J V 2003 N. Engl. J. Med. 349 145164

[36] Bissell M J and Labarge M A 2005 Cancer Cell 71723
[37] Bissell M J and Hines W C 2011 Nat. Med. 173209

[38] Lu P, Weaver V M and Werb Z 2012 J. Cell. Bio. 196395406

[39] Laperrousaz B, Jeanpierre S, Sagorny K, Voeltzel T, Ramas S,

Kaniewski B, Ffrench M, Salesse S, Nicolini F E and

Maguer Satta V 2013 Blood 122376777 